\title{
Linewidth influence in photonics logic device
}

\author{
A.P. Gonzalez-Marcos, T. Vivero and J.A. Martín-Pereda \\ E.T.S. Ingenieros de Telecomunicación. Universidad Politécnica de Madrid \\ Ciudad Universitaria. 28040 Madrid. Spain
}

\begin{abstract}
Photonics logic devices are currently finding applications in most of the fields where optical signals are employed. These areas range from optical communications to optical computing, covering as well as other applications in photonics sensing and metrology. Most of the proposed configurations with photonics logic devices are based on semiconductor laser structures with "on/off" behaviors, operating in an optical amplifier configuration. They are able to offer non-linear gain or bistable operation, being these properties the basis for their applications in these fields. Moreover, their large number of potential affecting parameters onto their behavior offers the possibility to choose the best solution for each case.
\end{abstract}

Keywords: logic device, optical bistability, linewidth, optical sensor, optical computing

\section{INTRODUCTION}

In all the logic structures proposed by our group, the basic behavior of the photonic logic device used is bistability. So we must analyze the behavior of our basic device in order to see afterwards how it influences on the more complex functions of the designed structure.

As we have already shown, (1) - (2), the linewidth of a single optical data signal, impinging onto the semiconductor laser amplifier, affects its hysteresis width as well as some of its more important properties, namely, on and off switching input light powers. This effect allows its application as a sensor for input light wavelength characteristics variations in any optical signal.

As a matter of fact, we have analyzed the bistable behavior of a semiconductor laser, operating in an amplifier configuration, with three different structures, namely, Fabry-Perot, distributed feedback or vertical cavity surface emission lasers. Commercial CAD software has been employed to study this effect in these structures. A functional model has been adopted in other cases. Some experimental results have been obtained too.

We have analyzed, with the help of these results, the influence of the hysteresis cycle characteristics on the behavior of some optical arithmetic units based in optically programmable logic cells. These cells were reported previously and analyzed by us (3) - (4), when they were working with Self-Electrooptic Effect Devices, SEEDs, as non-linear optical devices. The results are analyzed now with a more conventional device, namely, the above mentioned semiconductor optical amplifier.

In this work we will report the obtained results from the analysis on how the linewidth of the two optical signals applied to a logic device may affect to its expected performance. This new resulting behavior will directly affect to a more sophisticated unit, - as optical arithmetic units in optical computing or switch fabrics for optical communications - and, as a consequence, to their final behavior. In this case we will analyze the most well know and basic structure: a FabryPerot- Semiconductor Laser Amplifier working as a bistable device. The results may be extended to more complex architectures.

Photonic Materials, Devices, and Applications II, edited by Ali Serpengüzel,Gonçal Badenes, Giancarlo Righini Proc. of SPIE Vol. 6593, 659323, (2007) · 0277-786X/07/\$18 - doi: 10.1117/12.722036 


\section{BISTABILITY}

The Optical Bistability on Semiconductor Laser Amplifiers -SLA- under external light injection has been extensible study previously. It has been study for different types of SLA structures, as SLA-FP, SLA-DFB and, of course, for the most practice structure, due to its fabrication characteristics, VCSLA.

In this work we are presenting the result for the most known and easily modulated configuration: the SLA-FP. The physical phenomenon that causes the optical bistability in laser amplifiers is the dispersive nonlinearity, characterized by the existence of a resonator filled with a medium whose refractive index depends on the incident optical power. An increment in the optical power injected into a laser makes its resonant frequency to move towards longer wavelengths, due to the variation of the refractive index. This means that when an optical signal, detuned to the long wavelength side of the resonant frequency mode of the laser is injected into it, will cause, as a consequence of the variation of the refractive index of the active medium, the positive wavelength shift of the resonant emission frequency of the laser. Once a threshold is achieved the optical bistability can be observed. For a more detailed explanation of the optical bistability in laser amplifiers see for example (5).

As it is obvious, when a device has been fabricated, the internal parameters that affect the optical bistability transfer function cannot be modified for an specific external injected signal. However this transfer function can be adapted by the variation of optical input signal, since the optical bistability depends on the optical power injected and the wavelength of the external injected signal. Also, the bias current applied to the bistability SLA device will affect the final bistability function, which means there are at least three parameters to control the desire performance.

In our study we are assuming a constant temperature on the device, as a complete simulation model, including temperature, is not yet available. Fabry-Perot semiconductor lasers are well known but still it is needed some discussion on the different models to evaluate some principal parameters behavior as the linewidth enhancement factor (6). In an internal report we have compared the results obtained with several model (7)- (8)- (9) and results from the commercial software of VPI: we found no different between both of them. We have chosen VPI because, as a CAD environment, gives a large number of tools to deal with optical and electrical signals and a good device model library. One of the most important parameter to control bistability is the injected signal detuning of the resonance wavelength of the FP-SLA, as it can be seen on figure 1 .

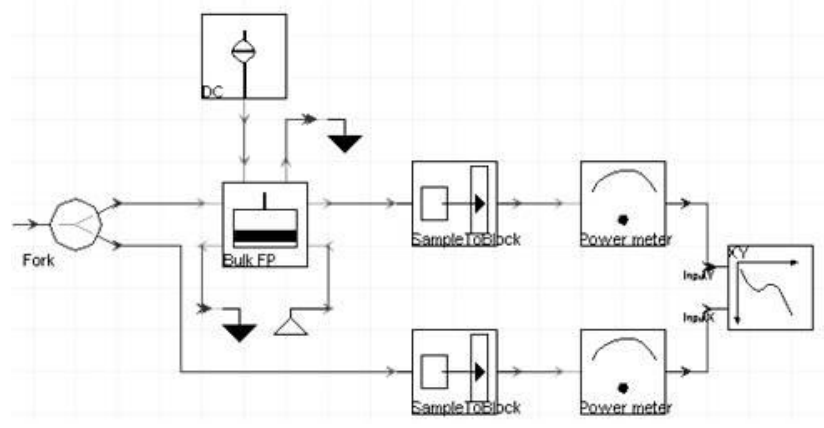

Fig. 1. Model diagram uses in VPI for transfer function. Optical Spectrum of FP-SLA output, without injected signal (tenuous resonant modes lines) and with $0,5 \mathrm{~mW}$ injected signal.

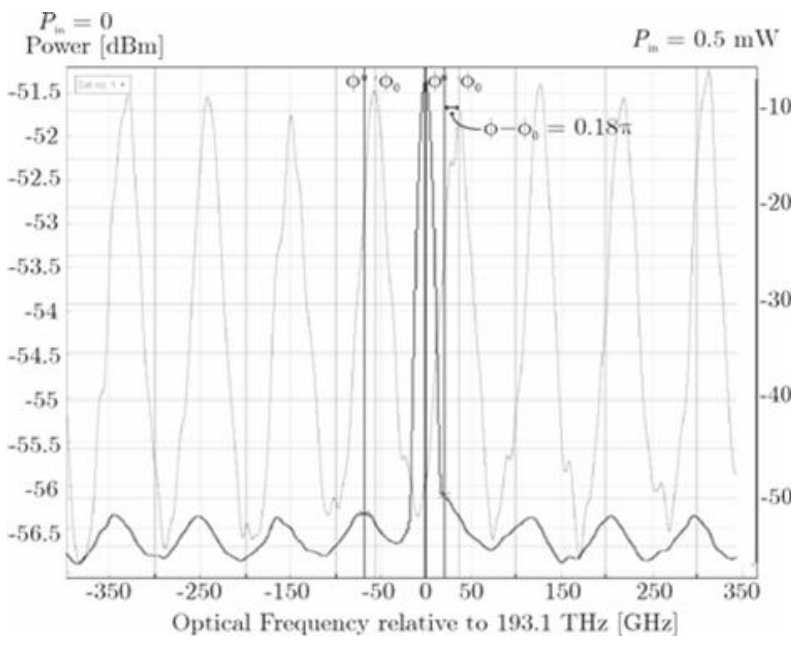

We have simulated the bistability for two wavelengths (second and third window). In both cases the wavelength chosen for the injected signal had a detuning of $20 \mathrm{GHz}$. The linewidth has been taken $\mathrm{i}$ as a wavelength parameter; on figure 2 , the bistability has been represented for an injected signal with a linewidth of $0,025 \mathrm{~nm}$. It can be seen that the function is different from the values of the parameters that characterize it, but in both cases has anticlockwise hysteresis cycle. The FP-SLA is different for each case; on Table 1 some of the parameters, as well as different values used for the model, are listed. Second window case shows a requirement of an optical power almost one order of magnitude smaller than with a $1500 \mathrm{~nm}$ signal. It is inferred from this fact that for any processing logic function it could better to use signals on 1300 $\mathrm{nm}$. In this way it would be less power consuming; but we cannot forget that until now the most developed technology has been based on $1500 \mathrm{~nm}$ due to optical communication applications. As both wavelengths seem to be relevant from different point of view, both of them are being study and presented in this paper. An extension to other optical frequency 
is obviously a must, but more from a fabrication device point of view than from a simulation approach, at least, at the stage of presentation of basic ideas as the ones presented in this paper.

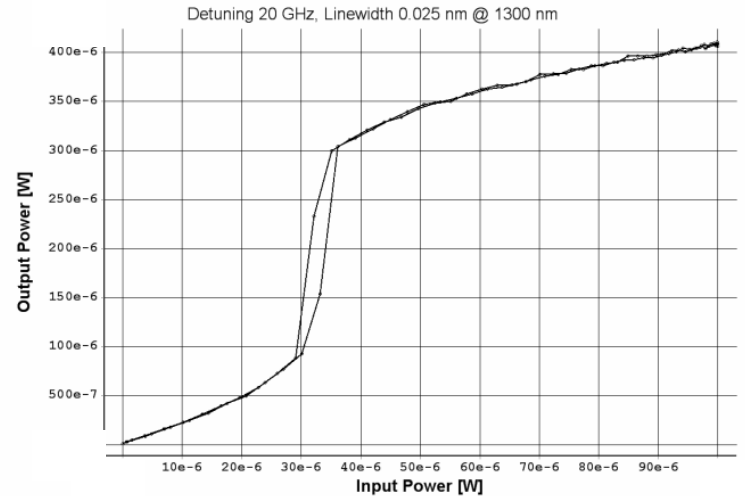

(a)

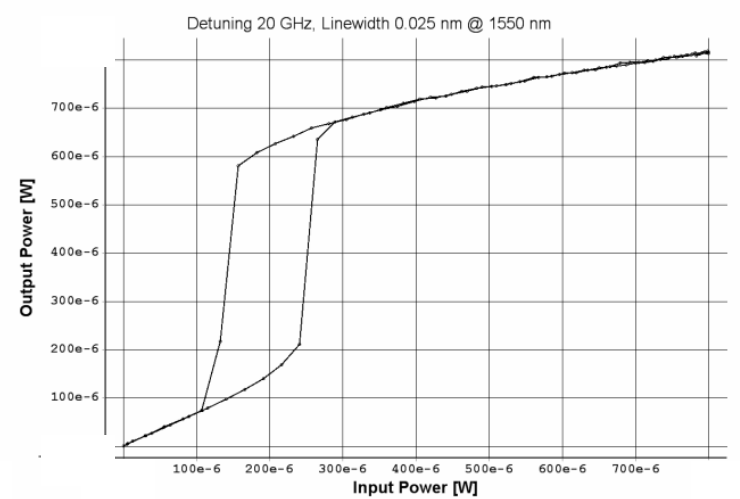

(b)

Fig. 2. Bistability (a) Second window; (b) third window. The axes represent optical power in $\mu \mathrm{W} . \Delta \lambda=0,025 \mathrm{~nm}$.

The output power from the FP-SLA it is also an important parameter because it will give the contrast ratio for an on-off device answer and it will determine the fan-out of the logic device.

\section{INFLUENCE OF THE INPUT SIGNAL LINEWIDTH}

The linewidth instability in a laser source is considered an inconvenient for any optical transmission due to signal distortions. Many sensors techniques to detect linewidth variation has been proposed and developed. The research group that present this paper has publishes one work [ (10)] based on the same behavior analyzed here. But in this case we are trying to use the same parameter, instant of a noise source, as a control parameter to fix the logic function to be performed by the optical logic device. Before we analyze this fact, we will study how the bistability anticlockwise function on the FP-SLA behavior changes in second and third window when the injected signal modifies only his linewidth value.
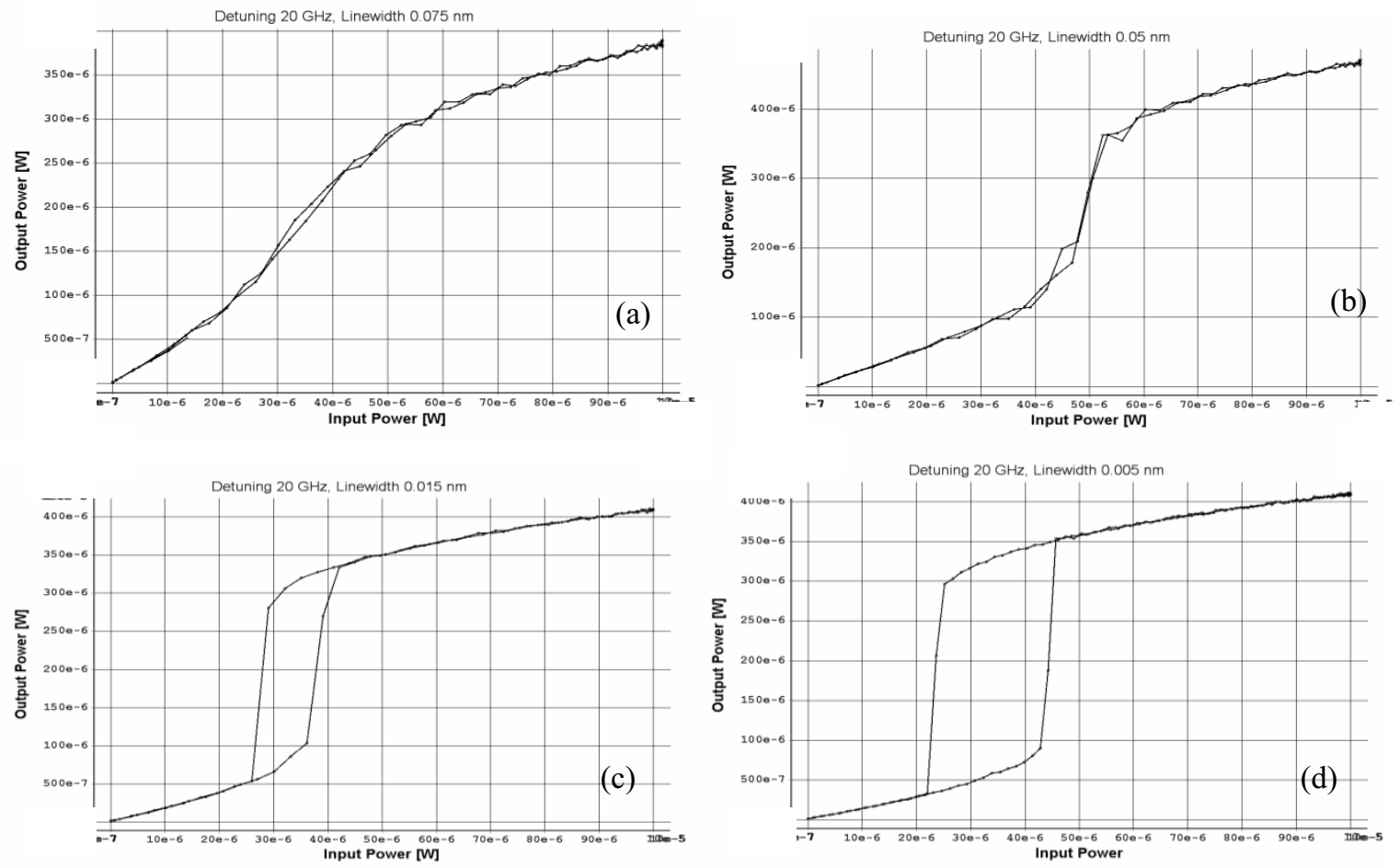

Fig. 3. Optical bistability FP-SLA $1300 \mathrm{~nm}$ and an initial detuning of $20 \mathrm{GHz}$ : a) $0,075 \mathrm{~nm}$; b) $0,05 \mathrm{~nm}$; c) 0,015 ; d) 0,005 
On figure 3 we can see the evolution of the transfer bistability function from a value of $0,075 \mathrm{~nm}$ to a more spectrally narrow optical signal. This performance demonstrates that if a clear bistability behavior is needed, and not just a nonlinear gain, it is a requirement to apply a highly monochromatic signal to the FP-SLA. This behavior can be expected as the physical phenomenon that causes the optical bistability in laser amplifiers is the dispersive nonlinearity. On figure 4, the same predictable evolution is demonstrated with an input signal with an initial detune of $20 \mathrm{GHz}$ for $1550 \mathrm{~nm}$.
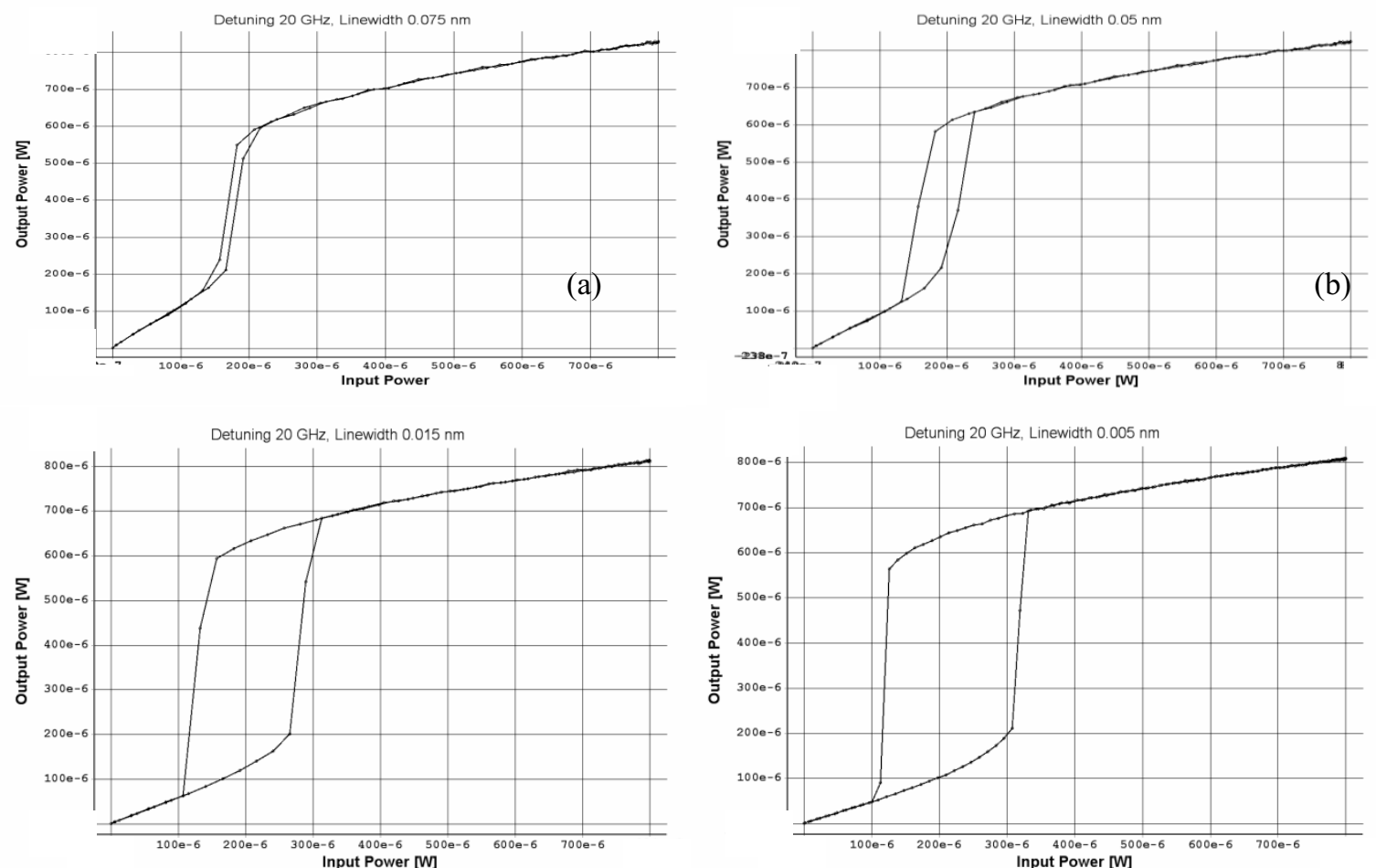

(c)

(d)

Fig. 4. Optical bistability FP-SLA $1500 \mathrm{~nm}$ and an initial detuning of $20 \mathrm{GHz}$ : a) $0,075 \mathrm{~nm}$; b) $0,05 \mathrm{~nm}$; c) $0,015 \mathrm{~nm}$; d) $0,005 \mathrm{~nm}$

The best way to represent the change evolution of the transfer function for different linewidth optical signal is by figures 5 and 6 . Figure 5 shows the linewidth versus hysteresis width, demonstrating a linear behavior which corresponds with the ideal response of a device with respect to a certain parameter. In this case we can use such a parameter as a sensor or as a parameter of control.

The hysteresis width it can be also analyzed from the point of view of switching on and off power. As the linewidth get smaller, the optical switching on power must be higher. The switching off has a smooth variation and is almost the same in every one of the cases presented here.

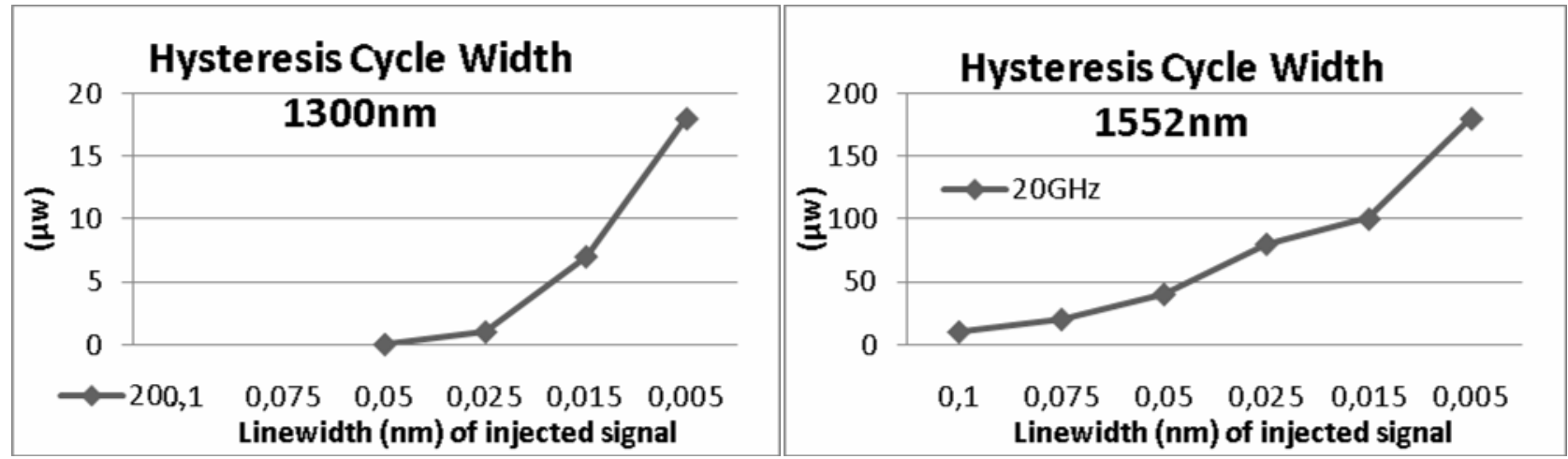

Fig. 5. Linewidth versus hysteresis width a) $1300 \mathrm{~nm}$; and b) $1550 \mathrm{~nm}$ 

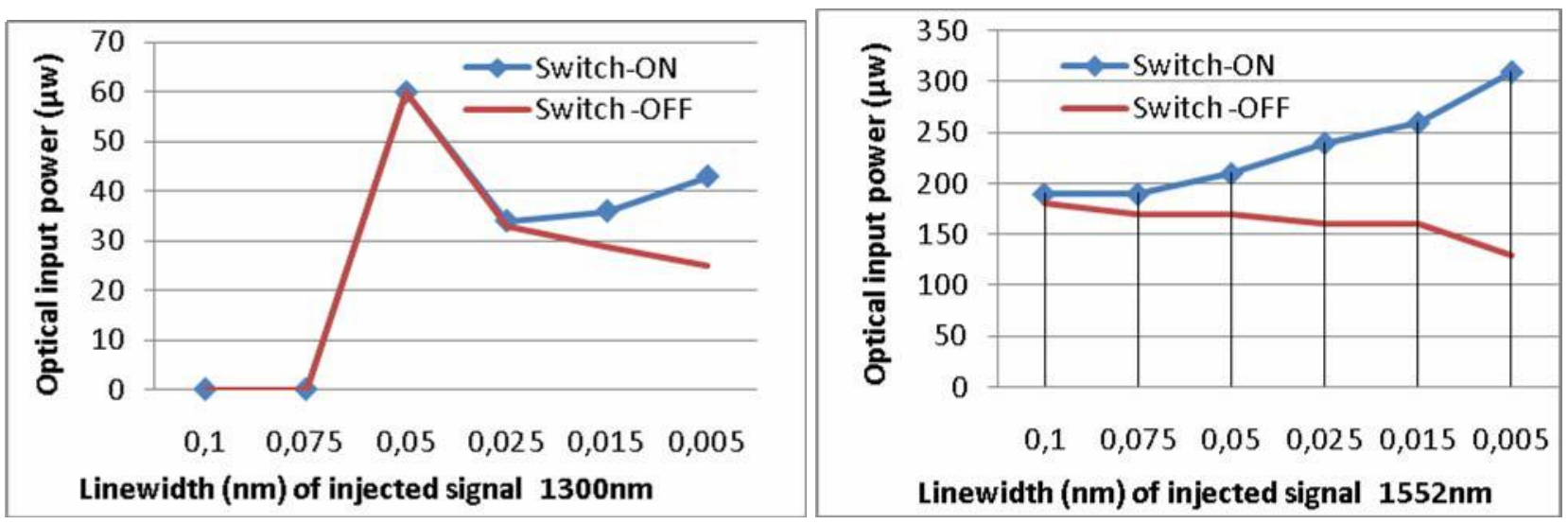

Fig. 6. Optical input power required to switch the output of the FP-SLA from a low value to a high value of optical power, or vice versa, with $20 \mathrm{GHz}$ detuning. a) $1300 \mathrm{~nm}$ - Optical output power: $100-300 \mu \mathrm{W}$; b) $1552 \mathrm{~nm}$ - Optical output power: $250-550 \mu \mathrm{W}$

The study of the dispersive optical bistability in semiconductor laser amplifiers has been carries out taking into account two parameters: the bias current applied to the device and the detuning existing between the resonant frequency mode of the laser amplifier and the injected signal. Our research group has study the VCSOA bistability and found a third parameter in this last case. This third parameter corresponds to the DBR- Distributed Bragg Reflector- period number of upper side of the VCSOA resonant cavity formed by two DBR and the study of it was reported in (11). In this work we are just considering a semiconductor laser amplifier with a Fabry-Perot cavity without DBR. Also we consider the device fixed on its manufactured aspect and on its bias working condition. As we consider the external signal as a variable we can also study if the previous behavior is maintained when the initial detuning changes. It is not our intention to extend this work to the study of this case but we can see on figure 7 how it is modified the linewidth versus hysteresis width. We can predict from it that, as higher is the initial detuning, more monochromatic must be the signal in order to obtain some bistability.

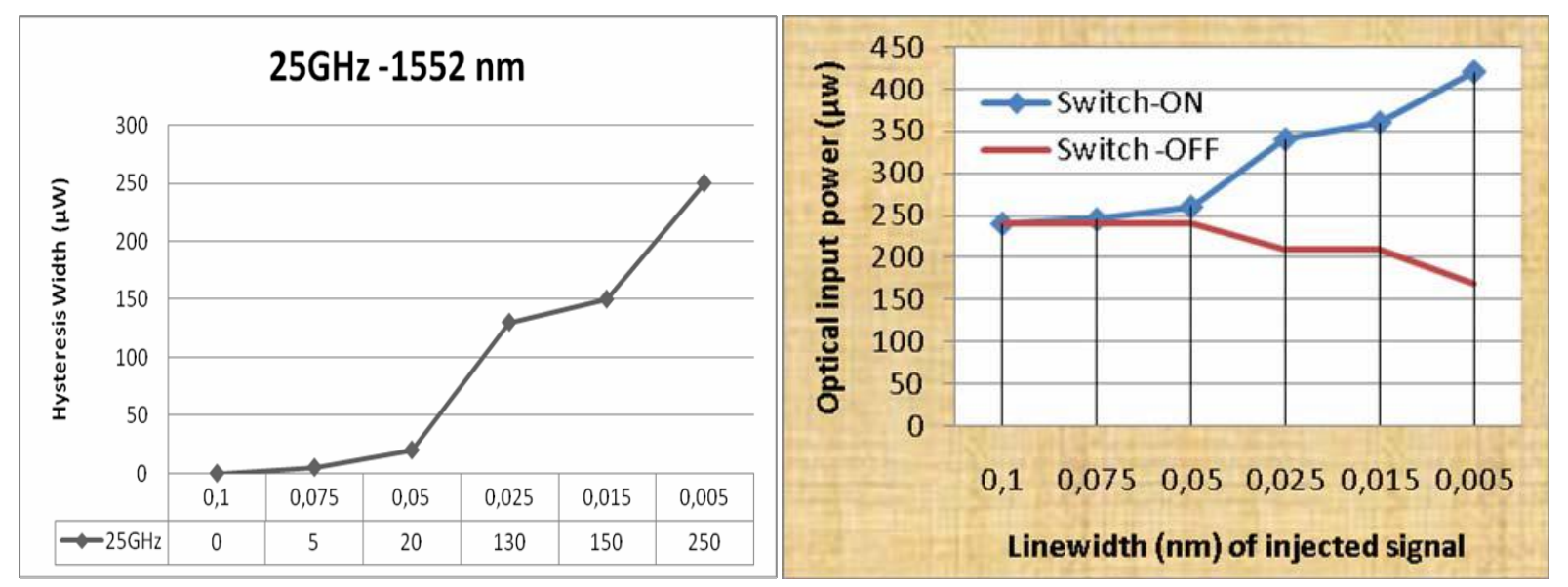

Fig. 7. $1552 \mathrm{~nm}$ - Optical output power: a) Linewidth versus hysteresis width; b) Optical input power required to switch the output

The previous study of the bistability loop was performed in conditions of small signal, which means we have not analyzed the time responses of our devices. We do not know what happen when the injected signal is a digital signal and we need a high bit rate. When we are thinking to apply this type of devices for optical computing, we must first think on digital processing. In this case, in order to take advantage of the best property of optical signal: the parallel processing; the classical computer architecture must be left apart. The authors have previously reported an optical programmable logic cell that has a different viewpoint from the classical or digital electronic counter part. In our solution, to obtain all the Boolean logic functions we apply a multilevel process instead of binary logic. The processing element has been described in (3). 


\section{LOGIC FUNCTIONS}

One of the basic structures for digital computer, in an Arithmetic Logic Unit (ALU), it is a half-adder, we have reported it (2) by simulation of the functional behavior with Matlab software tool. We study there how the tolerance of the devices used can produces an error on the sum of data. The error was a consequence of execute a wrong logic function.

The design of the half adder was done with the OPLC (Optical Programmable Logic Cell) by the well-know equations of a half adder with Boolean functions. The OPLC is based in two devices, called P and Q device. These devices have an hysteresis cycle which can be implemented with an optical bystable device. Since a few years we are working with this objective and some results has been obtained. It has been applying the optical bystability of semiconductor laser amplifiers (12), with different structures, FP-SOA, DFB-SOA, VCSOA (13) and some behavior analysts (14).

The Q device of the OPLC has an easy and well knows on-off transfer function, an equivalent transfer function of figure 2. In order to evaluate how the hysteresis cycle can affect in logic function let see on figure 8 the position of $d_{q}$, level of decision of the device, which it is crucial to perform the Boolean logic. The shift of this value changes the logic function, in one position the OR function is carry out and in another position the AND function is execute.

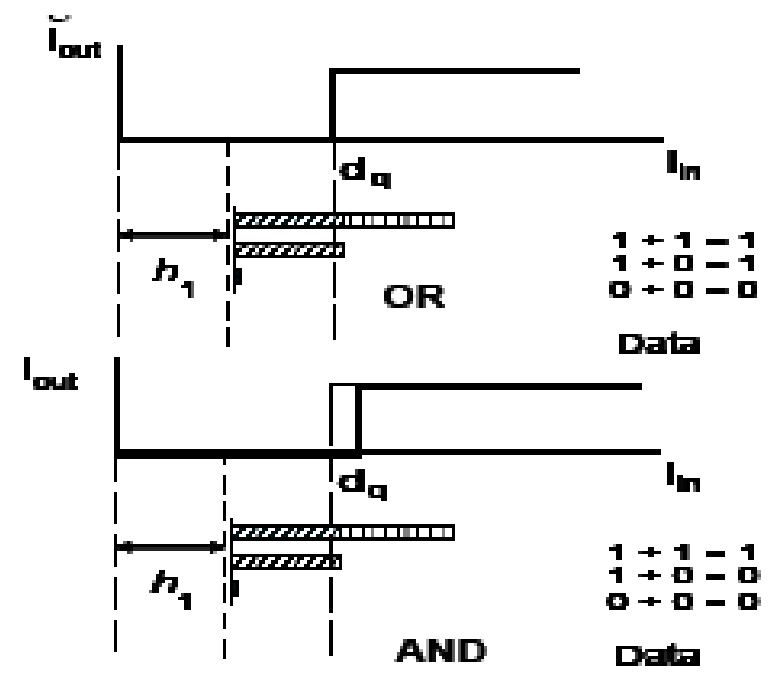

Fig. 8. Influence of the decision level on logic function implement in Q device.

TABLE I

Laser Parameter for the Fabry-Perot

\begin{tabular}{lc}
\hline Laser Param eter & FP1 \\
Cavity Length $(\mu \mathrm{m})$ & 350 \\
Left/Right Facet Reflectivity & 0.3 \\
Confinement Factor & 0.5 \\
Linear Material Gain Coeff. $\left(\mathrm{cm}^{2}\right)$ & $2.2 \cdot 10^{-16}$ \\
Linewidth Enhancement Factor & 6.9 \\
Linear Recombination Coeff. $(1 / \mathrm{s})$ & $1 \cdot 10^{8}$ \\
Bimolecular Recomb. Coeff. $\left(\mathrm{m}^{3} / \mathrm{s}\right)$ & $2 \cdot 10^{-16}$ \\
Auger Recombination Coeff. $\left(\mathrm{m}^{6} / \mathrm{s}\right)$ & $8.25 \cdot 10^{-41}$ \\
Transparency Carrier Density $\left(1 / \mathrm{m}^{3}\right)$ & $10^{24}$ \\
Fixed Internal Loss $(1 / \mathrm{m})$ & 5000 \\
Index Grating Coupling Coeff. $(1 / \mathrm{m})$ & - \\
Bias current & $0.8 \cdot \mathrm{I}_{\text {th }}$ \\
Frequency detuning (Ghz) & 30 \\
\hline
\end{tabular}

In this work we want to evaluate the problem of figure 8 from the point of view of optical bystable in semiconductor laser amplifier. We study first the most know and basic structure, the fabry-perot FP-SOA. We take one of the configurations that we have taken in account previously. The block diagram used to simulated the Q-device is on figure 1, the parameters apply are resume in Table I, and the bystability loop obtained is similar to figures 2 . In this case we are using $30 \mathrm{GHz}$ of detuning as it is show on Table I. 

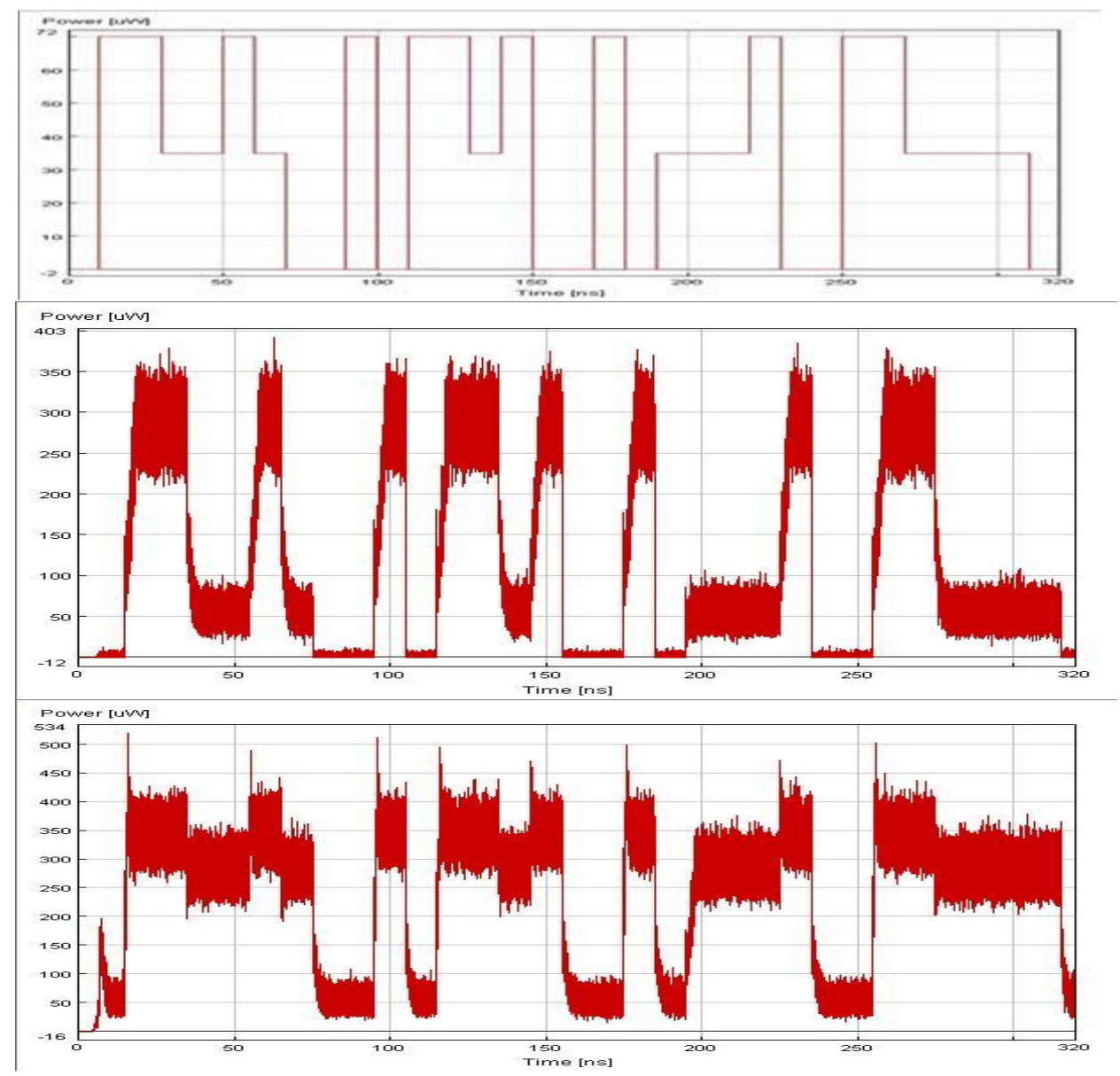

Fig. 9. Input data to the Q-device, AND function and $\mathrm{OR}$ function

Figure 9 shows the result obtained with the VPI software. As can be seen from the axis of the plot, data rate it is $100 \mathrm{MHz}$, It is not easy to use a higher frequency. It has been demonstrated that the carrier recombination time limits the high frequency response of optical bistability in laser amplifiers, but supposed to be higher than the bit rate used. Further analyzed on fabry-perot laser diode parameter of table I will give a better approach to a real device.

The bistability anticlockwise loop have been extensible study on his dependence with optical input power, initial detuning of optical injected signal and resonant frequency of FP-LD. As resonant frequency depends on bias current of FP-LD, the electrical current apply is another parameter to fix on the block model of figure 1. The way to change from AND to OR function on figure 9, depends on the relative position of switch ON and switch OFF optical power with respect the power of a bit -1 . The relative position can be change in different ways. Until know we have control this changes with a CW optical signal added to the data with an optical coupler Then the signal is still a three level optical power signal, but with different values in the input power. Output signal theoretically is binary an always with the same value.

Another way to control the relative position try to do not modify the amplitude of the input data signal, we can modify it with another parameters of the optical signal, which beside polarization is the wavelength or optical frequency. It is impossible to find a monochromatic source but we must think that there are many techniques to filter the optical signal to reduce his linewidth. So any of them, some based on non-linear effects, can be apply to control the linewidth of an optical signal modulated or not. If the amplitude of the injected signal to the bystable device is not change we must to change dynamically the bystablility loop. In this work we present the results controlling the logic function with the linewidth of the data signal or with another input signal with a different wavelength that produce a shift in the bystability anticlockwise loop and consequently modify the logic function to be executed by the Q device. 


\section{CONCLUSION}

We have reported the response of an optical bistable structure, namely single Semiconductor Laser Amplifiers SLA, when parameters of the input radiation change their values. The studied parameter is linewidth of the input light .We have shown that the output power offers a linear dependence with respect to this parameter in the case of single SLA working, in the transmisive mode. The same happens in the reflective mode, but it is not reported here. A computer model has been employed. The interest of this is their application to optical computing architectures. We have shown that changes in linewidth drastically affect to final response of the structure. Some considerations about the influence of this fact have been presented.

\section{ACK NOKLEDGEMENTS}

This work was partly supported by: Ministerio de Educación y Ciencia, grant FPI under project TIC 2003-04309 and grant TEC2006-13874-C02-02.

\section{REFERENCES}

1. Laser diode bistability as sensor of optical signal parameters. Gonzalez-Marcos, Ana P. and Martin-Pereda, Jose A. [ed.] John C. Armitage, Roger A. Lessard and George A. Lampropoulos. s.l. : Proceedings of the SPIE, 2004. Photonics North: Photonics Applications in Telecommunications, Sensors, Software, and Lasers. Vol. 5579, pp. 152159.

2. Method to analized the influence of hysteresis in optical arithmetic unit. González-Marcos, Ana and Martín-Pereda, José A. 11, s.1. : Society of Photo-Optical Intstrumentation Engineers, November 2001, Optical Engineering, Vol. 40, pp. 2371-2385.

3. Digital chaotic output from an optically processing element. González-Marcos, Ana and Martín-Pereda, José A. 2 , s.1. : Society of Photo-Optical Instrumentation Engineers, February 1996, Optical Engineering, Vol. 35, pp. 525-533.

4. Analysis of irregular bahaviour on an optical computing logic cell. González-Marcos, A. and Martín-Pereda, J. A. s.l. : Elsevier Science Ltd., 2000, Optics \& Lasser Technology, Vol. 32, pp. 457-466.

5. Kawaguchi, H. Bistabilities and Nonlinearities in Laser Diodes. s.1. : Artech House, 1994.

6. Bandwidth enhancement of Fabry-Perot quantum-well lasers by injection-locking. Jin, X. and Chuang, S. s.1. : Elsevier Science Ltd., june 2006, Solid-State Electronics, Vol. 50, pp. 1141-1149.

7. Rivas Moscoso, Jose M. Estudio del procesado fotónico con dispositivos biestables, principalmente en sus características "potencia de entrada-potencia de salida". Tecnología Fotónica - Grupo de Neurofotónica, E.T.S.I. Telecomunicación. 2005. p. 30, Private.

8. A qualitative comparison between two semiconductor laser amplifier equivalent circuit models. Lowery, A. J. 8, August 1990, IEEE Journal of Quantum Electronics, Vol. 26, pp. 1369-1375.

9. Computer Model of an Injection Laser Amplifier. Marcuse, D. 1983, IEEE Journal of Quantum Electronics, Vol. 19, pp. 63-73.

10. Vertical-cavity semiconductor optical amplifiers (VCSOAs) as optical sensing elements. Gonzalez-Marcos, A. P., Hurtado, A. and Martin-Pereda, J. A. [ed.] Goncal Badenes, Derek Abbott and Ali Serpengüzel. Belingham, WA : Society of Photo-Optical Instrumentation Engineers, 2005, Vol. 5840, pp. 262-271.

11. Modeling Reflective Bistability in Vertical-Cavity Semiconductor Optical Amplifiers. Hurtado, A., GonzalezMarcos, A. and Martin-Pereda, J. A. 3, March 2005, IEEE Journal of Quantum Electronics, Vol. 41, pp. 376-383.

12. An All-Optical Progrmmable Logic Gate with 1550nm Semiconductor Laser Amplifiers. Hurtado-villavieja, A. and González-Marcos, A. 2004. EOS Topical Meeting Optics in Computing . Vols. OIC-2004. ISBN 3-00-013069-1.

13. All-Optical Vertical-Cavity logic Gate. Hurtado, A., González-Marcos, A. and Martín-Pereda, J.A. SaintPetesburg : s.n., 2004. International Topical Meeting. Optoinformatics. ISBN: 5-7577-0157-7.

14. Bit noise with an optical logic gate with laser diode. Gonzalez-Marcos, Ana P., Martín-Pereda, Jose A. y Hurtado, A. [ed.] Peter Heszler. Gran Canaria : Proceddings of the SPIE, 2004. Fluctuations and Noise in Photonics and Quantm Optics II. Vol. 5468, págs. 56-65. 